\title{
Implementing Implementation: Practical Lessons Learned From Supporting Evidence- Informed Service Delivery in Community-Based Child and Youth Mental Health Agencies
}

\author{
Evangeline Danseco, Ann Barber, Kelcie Brown, and Charlie Carter \\ Children's Hospital of Eastern Ontario
}

\begin{abstract}
As child and youth mental health agencies apply the recommendations from the national mental health strategy, a strong focus has been placed on the implementation of evidence-informed practices to ensure the best outcomes for those they serve. Although a considerable amount of research exists on the implementation of best practices and the key factors to successful implementation, far less exists in terms of concrete strategies to support such initiatives. This article describes a unique initiative in Ontario that provided implementation supports and funding for agencies. Lessons learned and implications for bridging the gap between research and practice are explored.
\end{abstract}

Keywords: child and youth mental health, community-based agencies, implementation science, evidenceinformed practices

Evangeline Danseco, Performance Measurement Coach and former Director of Support Services, Ontario Centre of Excellence for Child and Youth Mental Health at the Children's Hospital of Eastern Ontario, Ottawa, Ontario; Ann Barber, Quality Improvement Specialist, Children's Hospital of Eastern Ontario, Ottawa, Ontario; Kelcie Brown, Research Assistant, Children's Hospital of Eastern Ontario, Ottawa, Ontario; Charlie Carter, Manager, Support Services, Children's Hospital of Eastern Ontario, Ottawa, Ontario.

Kelcie Brown is now at Queen's University and Charlie Carter is now at the Public Policy Forum.

The authors would like to acknowledge the participation of the project teams in the 14 agencies, especially the project leads who provided feedback for this article. We would also like to acknowledge all staff who contributed to this program, particularly Ian Manion.

Correspondence concerning this article should be directed to Evangeline Danseco, Ontario Centre of Excellence for Child and Youth Mental Health, 695 Industrial Ave. Unit 1, Ottawa, ON K1G 0Z1; phone (613) 737-2297 ext 3319; fax (613) 738-4894. Email: edanseco@cheo.on.ca 


\section{RÉSUMÉ}

Dans l'application des recommandations de la stratégie nationale en matière de santé mentale que font les organismes qui s'occupent de la santé mentale des enfants et des adolescents, on a beaucoup mis l'accent sur les pratiques fondées sur des données probantes, afin d'obtenir les meilleurs résultats possibles. S'il existe de nombreuses études sur la mise en œuvre de pratiques exemplaires et sur les facteurs clés liés à la réussite de cette mise en œuvre, il s'est fait beaucoup moins de recherche sur les stratégies concrètes utilisées pour réaliser ces divers projets. Cet article décrit l'un de ces projets, qui a permis de fournir à différents organismes ontariens du soutien et du financement dans ce domaine. On y explore les leçons que l'on peut en tirer et qui permettront de combler le fossé entre la recherche et la pratique.

Mots clés : santé mentale des enfants et des adolescents, organismes communautaires, science de la mise en œuvre, pratiques fondées sur des données probantes.

Changing Directions, Changing Lives was developed by the Mental Health Commission of Canada, with input from a wide range of stakeholders, and provides Canada with its national mental health strategy (MHCC, 2012). The third set of recommendations in the strategy focuses on providing appropriate, accessible, coordinated and effective community mental health services. These recommendations underscore the need for community-based child and youth mental health agencies to provide services that are based on the best available evidence.

In this article, we use the term evidence-informed practices to refer to the best available evidence. Evidence-informed practices (EIP) integrate empirical research with the experience and judgment of clinicians and the preferences of end-users such as children, youth, and families (Lomas, Culyer, McCutcheon, McAuley, \& Law, 2005; Ontario Centre of Excellence for Child and Youth Mental Health [OCE CYMH], 2013; Schlonsky \& Mildon, 2014; Starin et al., 2014). The EIP concept is consistent with the approach of the MHCC strategy in honouring the importance of the perspective of people with lived experience and underlining that community services should be in tune with local realities.

How can agencies that provide services to children and youth implement evidence-informed practices? What approach can be used to ensure that these practices improve clinical outcomes in a real world setting, are feasible, and are sustained over time? Implementation science offers a rigorous method to help ensure that community mental health providers are able to implement new practices within their organizations, with a view to ensuring fidelity to the practice, ongoing improvement, and sustainability (Aarons, Hurlburt, \& Horwitz, 2011; Fixsen, Naoom, Blasé, Friedman, \& Wallace, 2005). Training and professional development in and of themselves are insufficient to ensure a particular practice or program will be applied consistently and sustainably by providers in their organizations (Fixsen et al., 2005). Fixsen and his colleagues (2005) presented a framework to guide such an approach, and an application of this framework in the largest children's mental health agency in Ontario provided preliminary positive results (Barwick, Kimber, \& Fearing, 2011; Brown, Rounthwaite, \& Barwick, 2010; Kimber, Barwick, \& Fearing, 2012).

Building on this work, this article presents an innovative program that provided resources and support to enhance the capacity of community mental health agencies in Ontario to deliver evidence-informed practices to children and youth (MHCC, 2012, Recommendations 3.2.1 \& 3.2.4). The People Advancing Change 
through Evidence (PACE) program was delivered as a provincial initiative applying implementation science to address the needs of community-based agencies, and was not designed as a research project. Given that the program was a pilot with changes being made as the program was being implemented, a developmental approach to the evaluation was adopted (Patton, 2010). We gathered feedback on an ongoing basis and made adjustments along the way. We report on the perceptions of the agency project leaders on the successes, challenges, and lessons learned. The lack of client outcome data and limitations to the research methodology are due to the initiative's focus on tailoring supports to agencies, providing technical assistance, and making ongoing enhancements to the PACE program rather than standardizing methods.

We begin with a description of the program activities and components, including literature that informed the program development, implementation, and evaluation. We then present results from the participating agencies using mixed methods and discuss implications for implementing the mental health strategy within the current context in Ontario's child and youth mental health sector.

\section{PROGRAM DESCRIPTION AND SUPPORTING LITERATURE}

The PACE program was initiated in 2010 to bridge the research-to-practice gap among child and youth mental health agencies in Ontario. Consultations and focus groups were conducted with key stakeholders (e.g., members of our organization's advisory committee which includes youth, family members, researchers, and service providers), and with experts in implementation science and knowledge translation from Canada, the United States, and the United Kingdom. Their input helped identify a solution to the need.

The program used a phased approach to provide sufficient time for each stage of the implementation process based on the frameworks of the National Implementation Research Network (NIRN; Fixsen et al., 2005), the Replicating Effectiveness Programs from the Centers for Disease Control and Prevention (Kilbourne, Neumann, Pincus, Bauer, \& Stall, 2007), the Partnerships for Success model (Julian, Ross, \& Partridge, 2008; Kerns, Rivers, \& Enns 2009), the Interactive Systems Framework (Wandersman, 2009), and the Research in Practice model in the United Kingdom (Barratt \& Hodson, 2006; Hodson \& Cooke, 2007). Following the initial development of the program and accompanying tools, elements from other models in the published literature were also integrated, notably those from the Exploration, Preparation, Implementation, and Sustainment framework (EPIS; Aarons et al., 2011) and the Consolidated Framework for Implementation Research (CFIR; Damschroder, Aron, Keith, Kirsh, Alexander, \& Lowery, 2009). Figure 1 shows the various processes within each of the major phases of implementation: pre-planning, planning, doing, and sustaining.

The PACE program provided financial support to participating agencies for three years, from $\$ 25,000$ to $\$ 50,000$ each year. Agencies interested in participating in the program applied through a competitive process and participated in pre-planning activities. In the pre-planning phase, agencies conducted an organizational readiness assessment facilitated by staff from our organization. Topics covered in the assessment included the organization's leadership support for the implementation project, resources available to support the team and project, current training and professional development practices, the organizational culture relating to managing change, and most importantly the decision support system currently in place. In order to evaluate whether the new practice has contributed to improved clinical outcomes, there must be a system for accessing evidence and a culture of evaluative inquiry already in place (Botcheva, White, \& Huffman, 


\section{Figure 1}

\section{The PACE Program Framework for Implementing Evidence-Informed Practices}

\begin{tabular}{|c|c|c|c|}
\hline Pre-planning & Planning & Doing & Sustaining \\
\hline $\begin{array}{l}\text { - Ensure a decision } \\
\text { support system is } \\
\text { in place } \\
\text { - Assess } \\
\text { organizational } \\
\text { culture for change } \\
\text { - Identify initial } \\
\text { areas for } \\
\text { improvement in } \\
\text { clinical practice } \\
\text { - Obtain senior } \\
\text { leadership } \\
\text { commitment }\end{array}$ & $\begin{array}{l}\text { - Mobilize teams } \\
\text { and engage } \\
\text { stakeholders } \\
\text { - Develop change } \\
\text { management plans } \\
\text { - Assess client } \\
\text { needs, evidence, } \\
\text { and fit } \\
\text { - Develop } \\
\text { implementation } \\
\text { plans, and } \\
\text { communication } \\
\text { strategies } \\
\text { - Prepare } \\
\text { organizational } \\
\text { systems (e.g., IT, } \\
\text { HR, QI policies } \\
\text { and processes) }\end{array}$ & $\begin{array}{l}\text { - Obtain staff } \\
\text { training from } \\
\text { purveyors } \\
\text { - Obtain technical } \\
\text { assistance, } \\
\text { consultations, and } \\
\text { supervision with } \\
\text { purveyors } \\
\text { - Monitor and } \\
\text { evaluate processes } \\
\text { and preliminary } \\
\text { outcomes }\end{array}$ & $\begin{array}{l}\text { - Ongoing } \\
\text { monitoring of } \\
\text { implementation } \\
\text { drivers } \\
\text { - Ongoing evaluation } \\
\text { of outcomes } \\
\text { - Refine } \\
\text { organizational } \\
\text { policies and } \\
\text { processes relating } \\
\text { to implementation } \\
\text { - Document } \\
\text { adaptations to the } \\
\text { practice or program }\end{array}$ \\
\hline
\end{tabular}

2002). The assessment was followed by group discussions among the agency steering committee members and our program staff to clarify areas of strengths and needs.

During the course of the project in each agency, a relationship between our organization and the agency was established through a knowledge broker (Dobbins et al., 2009; Phipps \& Morton, 2013; Ward, House, \& Hamer, 2009). The knowledge broker acted as a consultant throughout the duration of the project, working with the teams to tailor the PACE program activities and to ensure agencies received consistent support. The knowledge brokers are master's level staff, with background and training on implementation science, evaluation, and quality improvement. Various models of knowledge brokering show support for this facilitated approach in scaffolding research and practice gaps (Bornbaum, Kornas, Peirson, \& Rosella, 2015; Dobbins et al., 2009).

We commissioned a series of e-learning modules for the knowledge base on implementation: leading change, teamwork, needs assessment, implementing EIPs, and monitoring and evaluation (Barwick, Boydell, van Dyke, \& Worling, 2010). These modules served as a guide for several activities in the planning phase. First, core teams across all agencies participated in sessions on leading change and teamwork spanning two days based on organizational learning and change management approaches (Austin \& Claassen, 2008; 
Botcheva et al., 2002; Brown et al., 2010; Edmondson, 2004; Hodson \& Cooke, 2007; Raghavan, Bright, \& Shadoin, 2008). Core teams were composed of individuals from all levels of the organization, including leadership, administrative, and front-line staff to ensure a high level of commitment throughout the agency. Second, to determine which practice would be implemented, agencies engaged with key stakeholders to conduct an in-depth review of clients' needs, the evidence on programs or practices that address those needs, and the fit of the practice with the organization. Assessing fit included considering long-term financial and human resource requirements. Third, core teams conducted an in-depth review of the implementation drivers (Fixsen et al., 2005) and the development of implementation plans. Implementation drivers refer to competency, organizational, and leadership components that impact the ability of staff to implement a practice or program (Active Implementation Hub, 2013). For example, knowledge brokers facilitated discussions of the implications of the EIP on hiring, selecting, and orienting staff. Fourth, the knowledge brokers facilitated sessions to identify the focus for the evaluation and helped develop the evaluation framework.

In the next phase, doing, staff training in the EIP was conducted at each agency by a purveyor or developer of the particular practice. Sessions relating to clinical supervision and ensuring fidelity to the practice or clinical approach were also conducted at this stage. These sessions ensured that the core components of the practice were being implemented and could be linked to client outcomes. Where needed, booster sessions on implementation topics were conducted by knowledge brokers.

In the final phase, sustaining, organizational policies and practices for the ongoing practice were updated or developed, including any scaling-up across the agency or to the wider community. Some agencies used an online sustainability tool to assess their readiness to sustain the new practice and identify priorities on which to focus (Luke, Calhoun, Robichaux, Elliott, \& Moreland-Russell, 2014). The final phase included a knowledge mobilization session where agencies shared their activities, tools, experiences, and lessons learned.

Throughout the phases, knowledge brokers met regularly with the agency teams to identify potential resources, facilitate discussions on the implementation drivers, and provide consultations to tailor PACE program activities. Knowledge brokers supported the teams in identifying where implementation science and organizational learning approaches could be applied to their unique organizational context. We coordinated regular meetings consisting of the project leads of each agency in each cohort, which met via teleconference at regular intervals.

\section{METHOD}

\section{Participating Agencies}

The PACE program was implemented in two cohorts. Eligible agencies for the program were those funded by the Ontario Ministry for Children and Youth Services, consistent with the mandate of our organization. In the first cohort, 12 agencies participated in the pre-planning phase in 2010, and six were identified through a peer review process as ready to proceed to the planning phase in 2011. In the second cohort, 13 agencies participated in the pre-planning phase in 2011 and eight were identified for the planning phase in 2012. All agencies who expressed an interest in participating in the PACE program submitted terms of reference and described their organizational practices on staff professional development and evaluation capacity. Applications were rated on the potential to improve mental health outcomes, the feasibility of the 
proposed project, and the readiness for implementation (e.g., the fit with agency strategic plans and with other provincial initiatives, use of decision support systems, or outcome measurement).

Fourteen agencies providing child and youth mental health services participated in the PACE program. A three-year model of the program from the planning to sustaining phases was used by 12 agencies. Two agencies used a two-year model where each phase was shortened to 6 to 8 months. Financial support for the first cohort of six agencies consisted of $\$ 50,000$ each year and $\$ 25,000$ each year for agencies in the second cohort to accommodate the participation of more agencies. All agencies contributed matching in-kind resources. Each agency assembled a core team comprising four to seven members and identified a project lead. Table 1 presents information on the participating agencies including the practice implemented and the context for implementing a particular practice or program.

\section{Procedures and Sources of Data}

Data was collected from participating agencies on an annual basis and upon program completion, and came from three sources: (1) an organizational implementation capacity checklist, (2) an online exit survey upon program completion, and (3) a final report.

An organizational assessment was completed annually to monitor progress in building the agency's capacity to implement and sustain their chosen EIP. For the first six agencies, the Checklist to Assess Readiness for Implementation (CARI; Barwick, 2011) was used. The CARI consists of 40 items across eight domains: organizational capacity, organizational climate/culture, staff capacity, implementation plan, system level capacity, functional considerations, senior leadership, and training. It was adapted from a tool proposed by Austin and Claassen (2008) which consisted of 20 items along four domains on a 4-point scale $(1=$ Not even close, 2 = Some way to go, $3=$ Nearly there, $4=$ We're there).

For subsequent years, our program adapted the CARI into an implementation capacity checklist (ICC) to incorporate elements of other supports provided by our organization (e.g., evaluation capacity building, youth engagement, and family engagement (Danseco, 2013). The ICC consists of 41 items across 6 domains (access and use of evidence; youth, family and community engagement; evaluation practices; learning culture; managing new/ongoing initiatives; training and implementation plan). In addition, the ICC included two open-ended items on the organizational strengths and challenges in implementing a new practice or program. The ICC uses a 7-point scale to capture greater variability in the stage of planning or implementation (from $1=$ In the parking lot, to $7=$ We're helping others get there). Copies of the ICC are available from the correspondence author.

Upon completion of the PACE program, project leads completed an online exit survey asking about their experience with the program and the implementation process. The survey consisted of 36 items in three categories (reflecting on PACE program supports, reflecting on outcomes, and reflecting on the future), using a five-point Likert scale ( $1=$ Not at all likely, to $5=$ Highly likely $)$. Each of the scales included an open-ended item and project leads responded to four additional open-ended items in two categories (student support and reflecting on the continuous learning journey).

Final reports from 13 of the 14 agencies participating in the PACE program were submitted one month following program completion. One agency has extended its implementation and will be completing the 


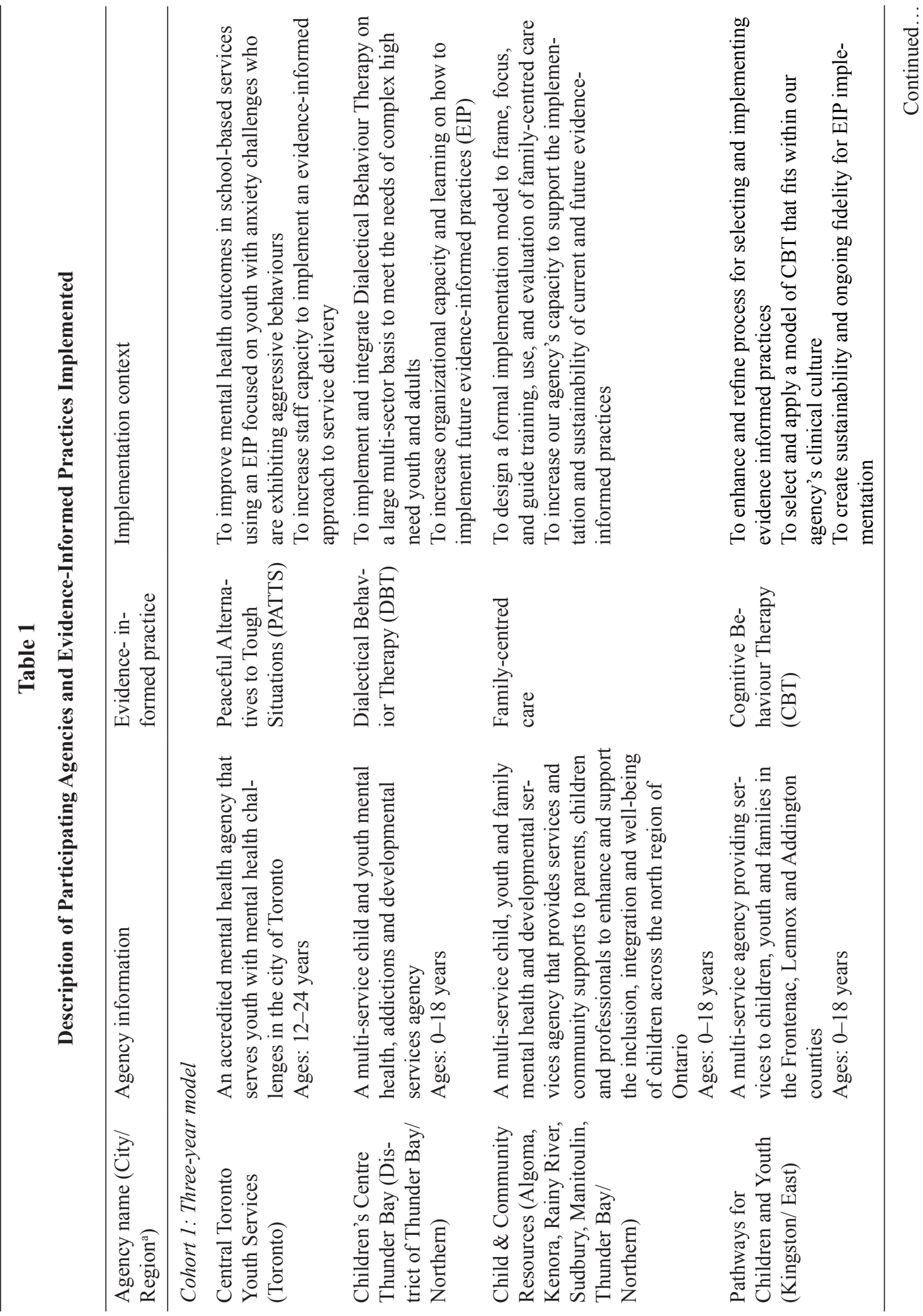



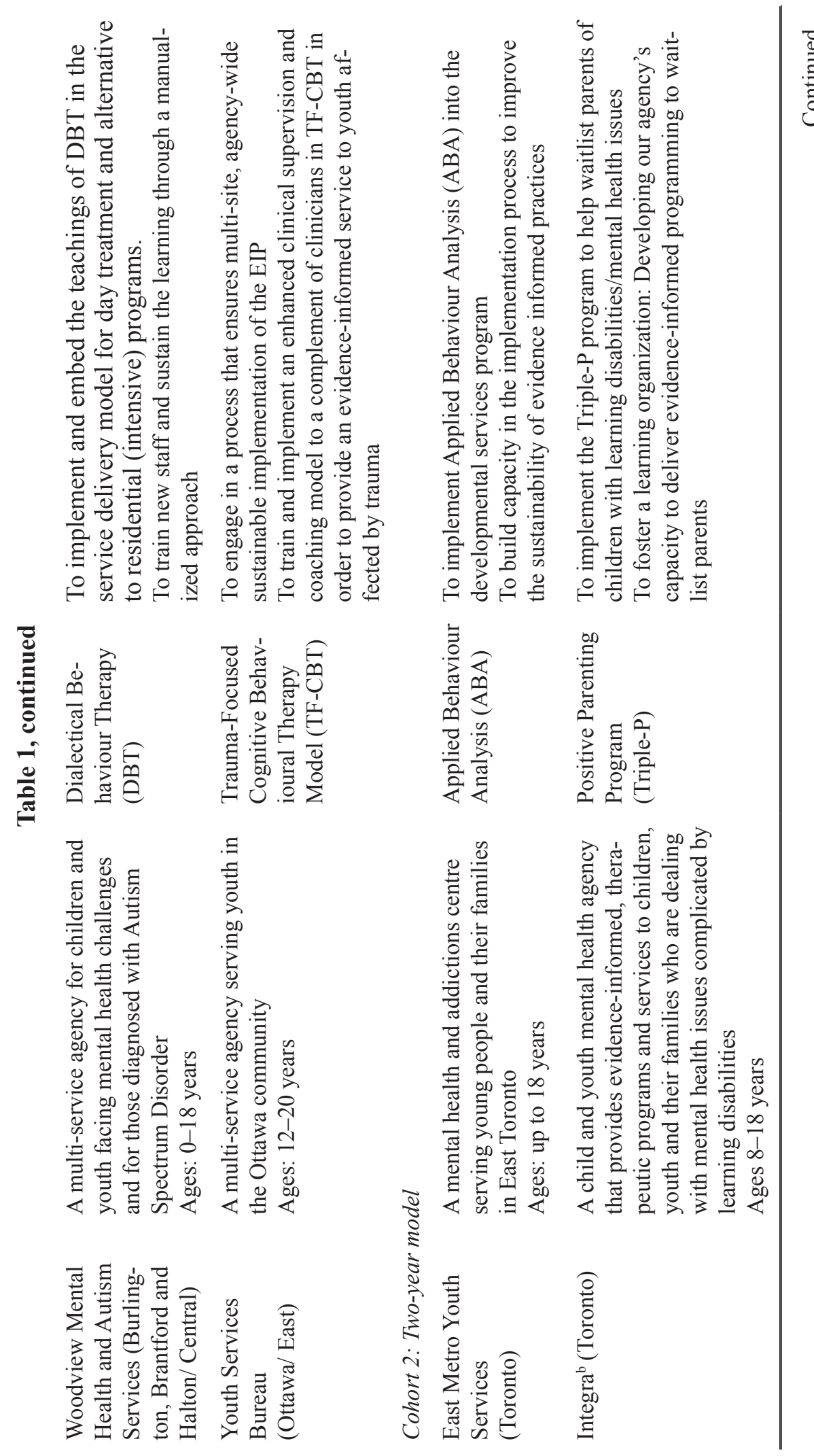

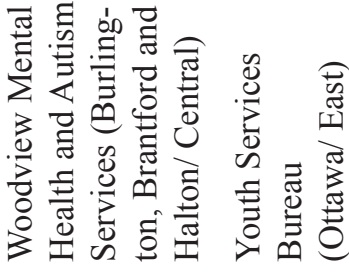




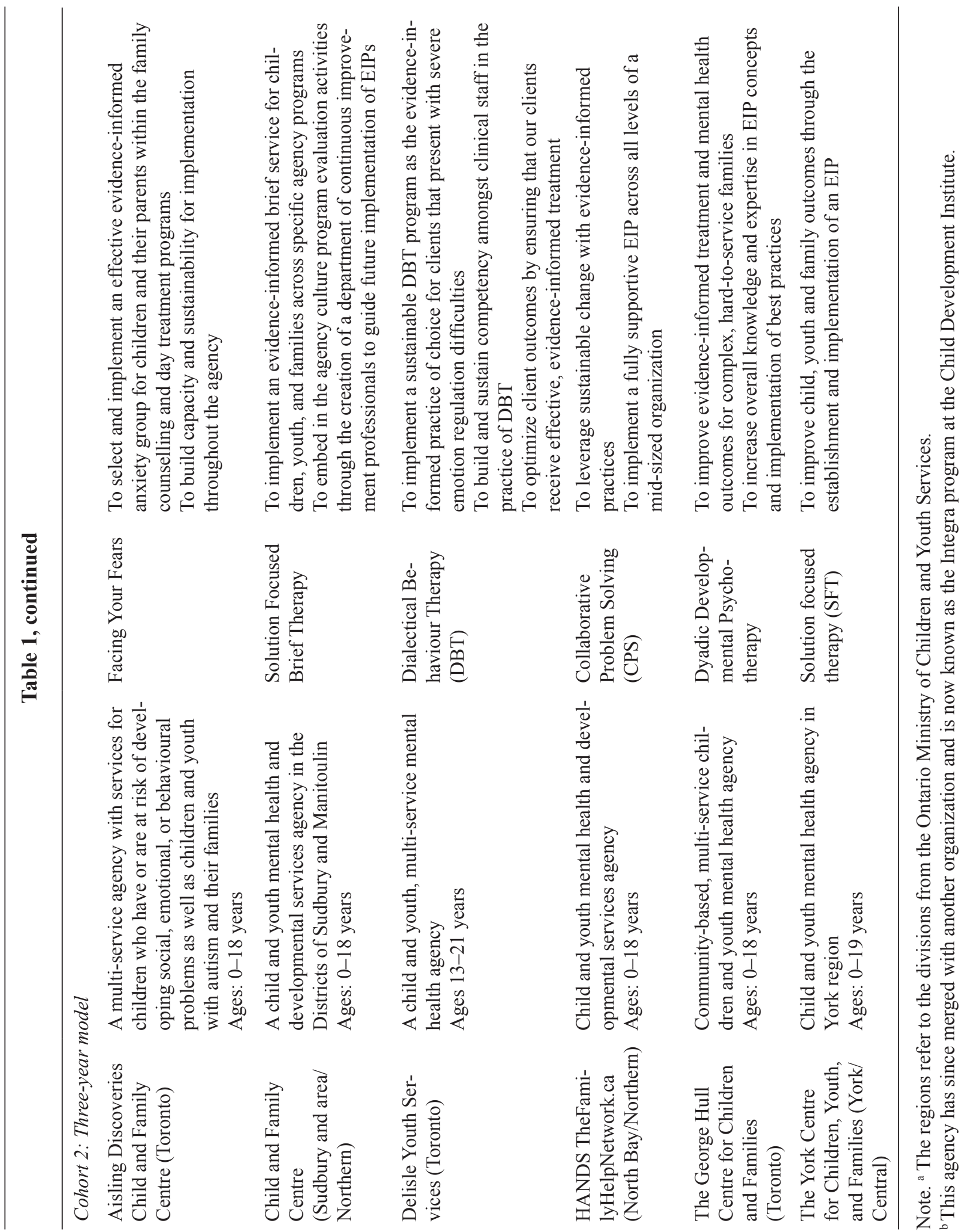


program by October, 2016. A final report template was provided to the agencies reflecting the stages of implementation, along with guidelines for writing about their activities, documenting the facilitators and challenges in the planning, doing, and sustaining phases as well as highlighting recommendations, lessons learned, and next steps. The final reports are publicly accessible documents (OCE CYMH, 2016).

\section{Ethical Considerations}

Given that the PACE program was not a research study and activities consisted of quality improvement efforts and program enhancements, formal ethics approval was not required. All agencies involved in the program gave their consent to participate in the program activities, as well as consent to share outcomes through a published paper. This consent was formally recognized through the signed support agreement between the agencies and our organization.

\section{RESULTS}

\section{Data Analysis}

Twelve agencies responded to the exit survey and submitted their final reports (92\% response rate). Quantitative items in the exit survey and implementation capacity checklists were summarized using Microsoft Excel 2016. Qualitative responses in the exit survey and the final reports were analyzed using NVivo qualitative analysis software (QSR International, Version 9, 2010). The first author coded all the qualitative data using the grounded theory approach and constant comparative technique, with discussions and reviews with the third author on emerging themes (Charmaz, 2006; Glaser \& Strauss, 1967). Following the initial inductive analysis and a review of the literature, the authors found that most of the themes corresponded to one group of constructs in the Consolidated Framework of Implementation Research (Damschroder et al., 2009; CFIR wiki, n.d.). The CFIR has five high level constructs (intervention characteristics, outer setting, inner setting, characteristics of individuals involved, and the implementation process). The implementation process construct consists of four sub-categories: (1) planning, (2) engaging, (3) executing, and (4) reflecting and evaluating. Hence, where relevant, the CFIR constructs and definitions were used, and when there were no corresponding CFIR constructs, new categories were created.

\section{PACE Program Supports}

Table 2 shows the means and standard deviations in the scales from the exit surveys for 12 of the 14 agencies. Based on ratings on the items and qualitative feedback, overall, agencies were very satisfied with the quality and content of the services provided throughout the PACE program. Agencies highlighted the value of having regular check-ins and guidance from assigned knowledge brokers. They also noted that comprehensive implementation training had a significant impact on the successful implementation of evidence informed practices. Teleconferences between the knowledge broker and the agency teams provided an opportunity to reflect on individual team progress and helped keep everyone informed about the progress of others, (e.g., information on strategies other agencies used for similar challenges, tools used for current processes). However, the teleconference updates convened to include all agencies were not as helpful. Several agencies saw value in the content of the e-learning modules but suggested it could have been more user friendly. The 
financial support from the PACE program was considered an essential resource that provided agency-wide training and was key to the successful implementation of EIPs. Agencies felt that structured PACE program activities such as facilitated sessions with knowledge brokers and annual submission of implementation plans and evaluation frameworks were critical to the successful implementation and sustainability of the EIP. These helped prioritize activities throughout each year and acted as a guide for the implementation process.

Table 2

Mean and Standard Deviation for Self-Report Quantitative Items on the Exit Survey.

\begin{tabular}{lc}
\hline Exit Survey Items & Mean (SD) \\
\cline { 2 - 2 } Reflecting on services (14 items) & $4.8(1.3)$ \\
Reflecting on outcomes (19 items) & $4.5(2.9)$ \\
Moving forward & \\
Continue to use an implementation framework beyond clinical training & $4.5(0.9)$ \\
Continue to enhance staff capacity in applying approaches from implementation science & $4.4(0.9)$ \\
Enhance organizational policies, practices and processes to support implementation activities & $4.3(0.9)$ \\
Mean for all items & $4.6(0.3)$ \\
\hline
\end{tabular}

Note. $N=13$ agencies. Items were rated on a 5 -point scale $(1=$ Not at all likely, to $5=$ Highly likely).

Eight agencies used some of the financial supports to hire undergraduate or graduate level students to assist in their implementation projects (e.g., conducting literature reviews, assisting in data collection or analysis). One agency said that involving students fostered connections with their local universities and will assist in future collaborations. Two agencies noted resource demands on staff time for mentoring, supervision, and health and safety requirements constrained the value of having part-time students engaged in their implementation activities. Agencies underscored the value of using funds to hire managers or coordinators devoted to the implementation projects throughout the two- or three-year period.

\section{Implementation Process}

Table 3 presents the themes that emerged from the qualitative analysis of the exit surveys and final reports, and focused primarily on the implementation process elements using the CFIR constructs. The column on lessons learned lists examples of practical activities or processes identified by the agencies.

A theme relating to the pre-planning process was added to capture the factors that contributed to an agency's readiness for implementation. The CFIR construct "readiness for implementation" aligned with themes we found in the pre-planning phase. The "implementation climate" CFIR construct was also added in the pre-planning phase.

As can be seen in Table 3, within each of the higher level categories, we found themes reflecting distinct activities. For example, in the Planning process, the activities of the PACE program helped provide guidance on team structures, who and how stakeholders will be engaged, and how to assess the fit of the practice with 
Table 3

\section{Major Themes Based on PACE Program Activities and Constructs in the Consolidated Framework for Implementation Research (CFIR)}

\begin{tabular}{|c|c|c|}
\hline $\begin{array}{l}\text { Implementa- } \\
\text { tion process }\end{array}$ & Brief description ${ }^{\mathrm{a}}$ & Lessons learned and sample processes \\
\hline $\begin{array}{l}\text { A. Pre-plan- } \\
\text { ning }^{\text {b }}\end{array}$ & $\begin{array}{l}\text { Assessing readiness for } \\
\text { implementation or "tangible } \\
\text { and immediate indicators of } \\
\text { organizational commitment in } \\
\text { its decision to implement an } \\
\text { intervention." (CFIR) }\end{array}$ & \\
\hline $\begin{array}{l}\text { Leadership } \\
\text { engagement }\end{array}$ & $\begin{array}{l}\text { "Commitment, involvement, } \\
\text { and accountability of leaders } \\
\text { and managers with the imple- } \\
\text { mentation." (CFIR) }\end{array}$ & $\begin{array}{l}\text { Consult with key staff, senior management and community } \\
\text { partners. } \\
\text { Present the initiative and obtain support at the board level. } \\
\text { Have a vision from senior management for program priorities, } \\
\text { financial resources and obtain their willingness to collaborate } \\
\text { at a systems level. } \\
\text { Where possible, obtain approval for long-term resources (e.g., } \\
\text { develop a five-year plan). } \\
\text { Align the proposed initiative with provincial or national efforts } \\
\text { (e.g., Ontario's comprehensive mental health and addictions } \\
\text { strategy). } \\
\text { Make links between the initiative and the agency's strategic } \\
\text { plan. }\end{array}$ \\
\hline $\begin{array}{l}\text { Available } \\
\text { resources }\end{array}$ & $\begin{array}{l}\text { "The level of resources dedi- } \\
\text { cated for implementation and } \\
\text { on-going operations, including } \\
\text { money, training, education, } \\
\text { physical space, and time." } \\
\text { (CFIR) }\end{array}$ & $\begin{array}{l}\text { Take account of costs for training, backfill for staff, costs of } \\
\text { any program manuals, consultation costs with purveyors, sub- } \\
\text { scriptions to evidence repositories. } \\
\text { Build-in costs from staff turnover. } \\
\text { Develop a five-year plan for resources and identify future } \\
\text { sources of funding. } \\
\text { Identify resource implications for multiple sites. }\end{array}$ \\
\hline $\begin{array}{l}\text { Access to } \\
\text { knowledge and } \\
\text { information }\end{array}$ & $\begin{array}{l}\text { "Ease of access to digestible } \\
\text { information and knowledge } \\
\text { about the intervention and how } \\
\text { to incorporate it into work } \\
\text { tasks." (CFIR) }\end{array}$ & $\begin{array}{l}\text { Obtain evidence from research literature to support the new } \\
\text { initiative. } \\
\text { Use the agency's website, shared drive, and internal e-library } \\
\text { for housing electronic information. } \\
\text { Ensure protected time for staff to access purveyors. } \\
\text { Ask purveyors and intermediary organizations for reading ma- } \\
\text { terials, resources, or connections with other leaders in the field. }\end{array}$ \\
\hline
\end{tabular}

Implementa- " "The absorptive capacity for tion climate change, shared receptivity of involved individuals to an intervention, and the extent to which use of that intervention will be rewarded, supported, and expected within their organization." (CFIR)
Adopt an evidence-informed approach to program development and improvement.

Identify competing priorities (e.g., preparing for accreditation) and leverage opportunities.

Tap into the agency's ways of sharing information (e.g., internal emails, shared learning sessions, "grand rounds").

Use previous experiences and staff expertise from other practices already implemented in the agency. 


\section{Table 3, continued}

Capacity for evaluation or quality improvement
Organizational processes and structures in place to assess, monitor, evaluate, reflect, and make ongoing improvements
Develop a culture which uses outcomes to inform services, and for operational planning and strategic change.

Use previous evaluation efforts to identify program improvements and areas for where to focus staff training.

Ensure that evaluation outcomes have led to program revisions, reflecting that the agency at both a management and front line staff level are committed to responding to what was learned. Promote positive experiences of evaluation or quality improvement among staff so they have an appreciation for evaluation. Build on previous evaluation activities that engaged partners and key stakeholders.

$\begin{array}{ll}\text { B. Planning } & \begin{array}{l}\text { "The degree to which a scheme } \\ \text { or method of behavior and } \\ \text { tasks for implementing an } \\ \text { intervention are developed in } \\ \text { advance, and the quality of } \\ \text { those schemes or methods." } \\ \text { (CFIR) }\end{array} \\ & \begin{array}{l}\text { Identifying the leaders and } \\ \text { meveloping } \\ \text { structures to } \\ \text { plan, execute, } \\ \text { evaluate and } \\ \text { sustain imple- } \\ \text { mentation } \\ \text { efforts }\end{array}\end{array}$

"The degree to which a scheme or method of behavior and tasks for implementing an intervention are developed in advance, and the quality of (CFIR)

Developing structures to plan, execute evaluate and mentation efforts
Identify the lead for the implementation project who has decision-making authority in the agency.

Consider hiring a manager dedicated to the implementation project to ensure dedicated and protected time for implementation activities.

Form a core team or steering committee for decision making and identify if an implementation team with staff representation is also needed.

Include representation from management, frontline staff, and other staff (e.g., research and evaluation, human resources, information specialist, family members).

Develop a presentation or information to the agency to introduce the team to the entire agency.

Build trust among team members through team-building exercises. 


\section{Table 3, continued}

Assessing needs and focusing implementation goals

Developing implementation plans

Engaging

Identifying and engaging key stakeholders
Identifying areas of strengths, needs, and priorities from various stakeholders that will be affected by the implementation initiative

Assessing implementation drivers, identifying priorities, and establishing key deliverables and timelines

\footnotetext{
"Attracting and involving appropriate individuals in the implementation and use of the intervention through a combined strategy of social marketing, education, role modeling, training, and other similar activities." (CFIR)
}

(CFIR categories: opinion leaders, formally appointed internal implementation leaders, champions, external change agents)
Identify the agency's areas of strengths, needs, and priorities in preparation for the initiative, using an organizational assessment tool.

Conduct an in-depth survey among staff to rate their knowledge, level of confidence and attitudes relating to the practice to be implemented.

Inquire about anticipated barriers and solicit ideas about resources they would find useful.

Consult with partners, families, and youth who will be affected by the new practice to understand their needs, and adjust implementation plans accordingly.

Consider delivering high-level presentations to engage staff and prepare them for change (e.g., introduction to evidenceinformed practices, overview of implementation science). Conduct a comprehensive literature review and identify practices that are the best fit for the client needs and agency context.

Build in sufficient time for planning, at least six months. Based on results of the organizational assessment and staff surveys, identify areas that require attention and prioritize early on.

Have detailed plans including transition plans brought about by staff turnover, to ensure continuity.

Find ways to address concerns about workload and burnout through open discussions and ongoing dialogue.

Ensure that implementation plans are integrated with other agency plans and initiatives (e.g., operational plan, service delivery plans, HR policies).

Spread out any competing projects to ensure that staff can focus energy in one direction. 


\section{Table 3, continued}

Additional categories: Youth and families

Intermediaries

Local university partners

Ongoing communications and knowledge mobilization
Include the youth and family voice throughout the implementation process.

Include the preferences and needs of youth, families and community partners in decision making.

Consider having intermediaries to guide the implementation process, make it educative and manageable, and to encourage reflection along the way.

Leverage resources from intermediaries to maximize the agency's limited resources.

Use intermediaries to provide implementation resources or connections with other experts.

Involve students in conducting literature reviews, preparing evaluation measures, collecting and analyzing data Foster connections with local universities for future evaluation or implementation of clinical services

Use existing regular meetings to provide updates on the implementation initiative and if possible, have the implementation initiative as a standing item (e.g., annual general meeting, internal newsletters, staff updates, meetings with community partners).

Organize special events focused on the implementation initiative (e.g., lunch and learn).

Consider developing a common icon for the implementation team in the email system to streamline communications.
C. Executing
“Carrying out or accomplishing the implementation according to plan.” (CFIR)
Conducting Delivering training on the new comprehensive program or practice through and flexible staff training

Identify staff who will be included in the training sessions and timelines.

Consider including senior management, staff from other programs, or levels of the organization to promote common language and understanding about the practice and mitigate risks from staff turnover.

Consider where possible a train-the-trainer approach. Identify a group of super-users or champions who will act as a team of experts on the practice.

Develop re-training sessions to account for staff turnover. 


\section{Table 3, continued}

Establishing supervision models and practices to support learning

Attending to logistics

Reflecting and evaluating

Enhancing capacity for evaluation and quality improvement
Developing and/or enhancing supervision models and practices to ensure fidelity to the practice or program being implemented, to support ongoing staff learning through peer support or communities of practice

Ensuring implementation activities are organized with attention to geographical considerations, accessibility of training events, and availability of resources and staff

"Quantitative and qualitative feedback about the progress and quality of implementation accompanied with regular personal and team debriefing about progress and experience." (CFIR)

Enhancing the knowledge and skills of key staff and of the organization to conduct evaluation and ongoing improvements
Encourage staff to bring questions and concerns to clinical supervisions with managers and during team meetings in order to build staff capacity and ensure practice fidelity.

Appoint a clinical lead for the new practice being implemented, with a scheduled and dedicated time for meeting with staff on

the new practice, and time for knowing current literature on the practice.

Have a dedicated training on supervision for clinical managers. Obtain coaching sessions with the purveyor.

Outline skill requirements for the practice and use these as a framework for supervision meetings and further staff development.

Use case review meetings to discuss client processes, solidify knowledge gained and enhance learning that has been developed during practice.

Consider pairing a novice with experienced staff so that new staff can benefit from the knowledge learned and avoid some of the pitfalls.

Consider supporting a community of practice from various teams or sites.

Include night, respite, casual, or part-time staff in the training, communications, and key implementation activities.

Use technology for training or communications with multiple sites.

Identify a central resource that staff can access, including electronic resources.

Consider providing a complete set of printed resources and manuals in each site.

Develop a program logic model and evaluation framework, to focus on what aspects to evaluate and plan for next evaluation activities.

Share evaluation findings with staff to increase their motivation.

Use program evaluation to shift the organizational culture towards learning. 


\section{Table 3, continued}

$\begin{array}{ll}\text { Monitoring } & \text { Obtaining and using informa- } \\ \text { and evaluating } & \text { tion about any aspect of the } \\ \text { implementa- } & \text { implementation process to } \\ \text { tion efforts } & \text { make course corrections or ad- } \\ & \text { dress issues }\end{array}$

Monitoring and evaluating fidelity to the selected practice or program

Monitoring and evaluating organizational implementation capacity
Monitoring and evaluating changes in service delivery
Assessing the extent to which staff are implementing the core components of the practice or program

Assessing changes in organization's capacity to implement the practice or program
Use evaluation findings and needs assessment results to identify which aspects to pilot or begin with.

Review processes to see what's working, what may have been missed and what requires adjustment.

Re-visit the implementation plan and identify areas that were skipped or any gaps in the process.

Review tools and measures and make necessary adjustments based on staff and client feedback.

Ask staff for their feedback on the initiative before and after receiving updates about the project.

Ask staff if they feel they have received sufficient training and identify areas where they need further information or training. Conduct a survey to determine the increase in staff's level of knowledge pre- and post-training on the practice. Identify a fidelity checklist from the purveyor or from other agencies implementing the practice.

Discuss with staff the areas where fidelity is not being met, identify reasons and potential solutions.

Use a community of practice to address questions about the practice and enhance fidelity.

Ensure there is sufficient information from both process and outcome evaluations to make informed decisions on replication or scaling up.

Share knowledge learned through the implementation to the entire agency as a form of internal capacity building.

Review results from the implementation capacity checklist and identify areas for growth.

Revisit terms of reference and identify gaps or challenges, and any organizational processes that need to be changed or updated.

Share challenges, successes and learn from the experiences of others.

Incorporate common implementation language and constructs when building "home-grown" programs.

Assessing improvements in delivery of programs or services that are directly impacted by the newly implemented practice or program
Review the possibility of having more groups or sessions from wait lists or availability of resources.

Identify any changes needed for referral processes and information, particularly for updated information to external partners.

Revamp processes on individual parent meetings, reporting guidelines, or program timelines where needed.

Identify any needed changes in youth satisfaction surveys or youth perception of care. 


\section{Table 3, continued}

Monitoring Assessing improvements in and evaluating clients' clinical outcomes, client outcomes including perceptions of care and services
Make sure that clients referred to the program meet criteria. Create a dashboard to support the data analysis of all measures. Compare results of client outcomes with results from previous or usual practice.

Identify areas where there has been no or little improvement, and identify potential reasons and solutions.

Share information to clients and staff, and celebrate successes.
D. Sustaining and spreading

Strengthening organizational policies and processes relating to implementation drivers
Spreading implementation capacity
Activities or processes to scaleup the practice or implement other new practices

Developing or refining organizational policies and processes relating to staff selection, orientation, performance management, training and supervision

Review alignment with the agency's strategic priorities.

Create an agency-wide EIP implementation committee to support policies and procedures on staff training and implementation of new practices.

Review the practice within the broader continuum of care and update relevant materials or processes.

Develop and/or revise existing staff orientation manuals to incorporate implementation activities and clinical principles. Create documents that can be accessed by all staff (e.g., on intranet, Sharepoint).

Develop detailed templates or documents such as administrative procedures for outcome measures, templates for weekly session notes, session fidelity measures.

Develop policies on the use of copyrighted materials from purveyors.

Update documents so that staff who request professional development training include an implementation framework.

Utilize the teams who participated in the core team or steering committee for new implementation work.

Use the PACE program model and NIRN frameworks for implementing new practices or programs.

Share the hexagon tool, implementation plan, evaluation plan to groups within the agency or with external partners who are planning to implement new practices.

Apply the implementation framework to scaling-up existing "home-grown" programs.

Use program logic models and process mapping to ensure clarity and accountability.

Engage clinical and administrative leads on next steps for enhancing implementation of new practices in the agency.

Continued... 


\section{Table 3, continued}

Leveraging Activities to ensure ongoing resources available resources including collaborations with external partners to maximize resources
Consider reconfiguring funding allocations across programs. Consider requesting clients for contributing a nominal fee to offset costs for manuals or workbooks.

Pool resources with other agencies in combining training or consultations with the purveyor.

Compile a directory of who's implementing what. Ensure that intermediary organizations make implementation resources and tools available and accessible.

Note. ${ }^{a}$ The descriptions with CFIR denote that these are currently in the CFIR framework while those with no CFIR are categories that emerged from our qualitative analysis.

'The "Readiness for implementation" is currently classified in the "Inner Setting" in the CFIR Framework, and consists of leadership engagement, available resources, and access to knowledge and information. "Implementation climate" is a category in the Inner Setting.

client needs and agency context. Activities with implications for sustaining also need to be integrated in the Planning process, particularly to address staff turnover, which all agencies identified as a challenge. This is particularly important as it has implications for sustainability.

For the Engaging process, the CFIR constructs lists stakeholder types. We found other stakeholders not explicitly mentioned in CFIR such as youth, families, purveyors, and intermediaries. More importantly, themes on the mechanisms for engaging stakeholders emerged. For the Executing process, three themes emerged, with clinical supervision as a major consideration. All agencies indicated the challenges in addressing staff turnover and in resource constraints; hence, supervision was a critical component for ensuring ongoing sustainability and fidelity to the EIP and for obtaining quality outcomes. For the Reflecting and Evaluating process, several areas emerged relating to both process and outcome evaluation, as well enhancing evaluation and implementation capacity of the organization.

The last process, the Sustaining and Spreading process, is not a CFIR construct, but rather part of the PACE program itself. Activities to sustain the practice begin at the planning stage, and the focus at this stage was on updating or revising organizational policies and consolidating implementation processes so that the organization was ready to generalize to other practices that will be implemented. The supplementary table provides detailed examples of new tools, activities or processes to promote Sustaining and Spreading. 


\section{Supplementary Table}

New Tools, Activities, and Processes That Emerged From Implementation of Practices in the PACE Program

\begin{tabular}{|c|c|}
\hline Categories & Sample Tools, Activities or Processes \\
\hline Additional funding & $\begin{array}{l}\text { Fundraising department seeking funding from private partners } \\
\text { Collaborating with other agencies to share resources and funding }\end{array}$ \\
\hline Client process improvements & $\begin{array}{l}\text { Verifying client fit to the program using standardized measures, not relying on } \\
\text { subjective opinion from intake workers or clinicians } \\
\text { Adjustments made to the referral process } \\
\text { Additional section added to the youth satisfaction survey to track Dialectic Behav- } \\
\text { ior Therapy (DBT) outcomes } \\
\text { Revamping individual parent meetings } \\
\text { Realigning reporting guidelines } \\
\text { Adjusting program timelines and introducing a number of assessment and fidelity } \\
\text { measures } \\
\text { Creating templates that have dictated new report writing practices }\end{array}$ \\
\hline Effective training methods & $\begin{array}{l}\text { Pairing new staff with experienced leaders. This has allowed the newer staff to } \\
\text { benefit from the experienced leaders' knowledge and avoid some of the pitfalls. } \\
\text { Establishing a DBT therapist email list and face-to-face meetings organized two } \\
\text { times per year } \\
\text { Developing in-house training as a means of continuing to build on the internal } \\
\text { capacity to implement and sustain }\end{array}$ \\
\hline New organizational structure & $\begin{array}{l}\text { Creating a department of professionals dedicated to evaluation activities support- } \\
\text { ing all services in ensuring informed decision making based on measurable data } \\
\text { and evidenced informed technique. } \\
\text { Launching a new internal Intranet. }\end{array}$ \\
\hline Staff performance & $\begin{array}{l}\text { The core team has reverted to being a professional development committee, with a } \\
\text { new mandate to teach and coach staff when they request training in a new model, } \\
\text { as to how to plan, implement, evaluate, and sustain the practice using the PACE } \\
\text { program framework } \\
\text { Creation of an evidence-informed practice (EIP) implementation committee, } \\
\text { tasked with supporting the agency in integrating and implementing EIPs. We have } \\
\text { also developed new policies, procedures and training to support these practices. } \\
\text { Creation of an EIP implementation orientation booklet to help support staff in } \\
\text { learning about implementation and program development. } \\
\text { The core team has developed a DBT Framework outlining skill development } \\
\text { requirements for staff delivering different components of DBT. This framework } \\
\text { is used in clinical supervision to review learning strengths and areas for further } \\
\text { development. } \\
\text { Continuing to develop an orientation package for new staff and a flow chart that } \\
\text { will create a pathway for staff to follow as they begin treatment with a new client }\end{array}$ \\
\hline
\end{tabular}

Continued... 


\section{Supplemental Table, continued}

Tools

\section{Implementation Outcomes}

Given that the CARI had been the original assessment used for the first six participating agencies before the shift into the ICC, only seven agencies had complete data for comparison purposes (54\% of 13$)$. In general, there was a trend towards an increase in scores in all domains across three years for the five agencies in the three-year model and for the two agencies using the two-year model. The mean score in the ICC for the first year was 4.0, 4.6 in the second year, and 5.4 in the third year out of a possible score of 7. Due to the small sample size, further analysis to determine significance was not appropriate.

The scale mean for perceived outcomes by project leads in Table 2 indicates a relatively high score. For example, the mean in the item, "Develop a stronger understanding of how to implement evidence-informed practices" was 4.9 (s.d. $=0.3$ ) out of a possible score of 5. Similarly, the mean rating for the item, "Increase our ability to scale up a practice to other programs in our organization or community" was 4.7 (s.d. $=0.5$ ). Overall the high scores indicate that participants believed that participation in the PACE program helped them to develop a stronger understanding of how to implement EIPs, as well as scale up other programs in their organization. Their participation enhanced their evaluation knowledge, skills, and partnerships with other organizations. Identifying available resources locally and beyond, along with strengthening existing partnerships in the sector, were identified as challenges for some participants.

From the qualitative data, organizational outcomes included enhanced evaluation knowledge and skills, enhanced partnerships with other agencies, increased staff capacity for implementing the target EIP, and enhanced client outcomes. More importantly, agencies reported the use of the lessons learned in their 
current work as helpful in implementing other EIPs or scaling-up the EIP. For example, one agency has used a process of assessing fit of a practice to their client population, staff and context for its programs that transition youth to the adult mental health system, and programs for their LGBTQ services.

Two overarching themes were predominant: (1) the importance of comprehensive planning for various processes that provided flexibility to address unforeseen challenges, and (2) the importance of engagement and ongoing communication with a broad range of stakeholders. Moreover, participating agencies highlighted several mind shifts in relation to their approach to implementation based on their current work. For example, several agencies noted the realization among core team members and staff that implementation is a complex ongoing process with many components, and that it takes more time than expected. One agency summarizes the lessons learned as follows:

The cumulative experience of implementing an intensive and complex intervention has required significant resources (both staff time and money) to orchestrate. It has clearly had a formative impact on our respective organizations with many lessons learned including:

- Critical importance of careful and thoughtful planning prior to embarking on any training or service delivery change.

- Need to engage key stakeholders both within each organization and across community stakeholders to achieve a shared collaboration and ownership of implementation.

- The importance of creating a vision of "why" the implementation plan and intervention is needed.

- The value and necessity of a steering group which includes "champions" while simultaneously having the capacity to complement each other in terms of skill sets and responsibilities.

- The need for flexibility and ability to adjust plans as circumstances change.

- Provision of comprehensive training and active learning strategies for therapists.

- Recognition of the challenges involved in sustaining any implementation project and that sustainability requires ongoing attention and effort to avoid treatment drift or drop in services. (Schmidt, 2015).

\section{Client Outcomes}

As noted above, the PACE program did not directly collect information on child, youth, and family outcomes, given that each agency selected the practice that met their needs. Participating agencies reported preliminary client outcomes as a result of their implementation projects. Some agencies noted positive prepost improvements in client functioning (e.g., Dermenjian, et al., 2016; Grant, Sears, Tsagarakis, \& Rolfe, 2015), increased satisfaction with services and improvements in client perception of care (e.g., Anderson, 2016). Also of significance, many acknowledged the enhanced partnerships developed with youth and fam-

ilies (e.g., Grant et al., 2015). Agencies noted that involving youth, families, and partner agencies needs to be included throughout the implementation process. 


\section{DISCUSSION}

Implementation science has been heralded as a solution to bridging the research-practice gap particularly among human service organizations (e.g., Aarons et al., 2011; Fixsen et al., 2005). Most of the existing literature on implementation among community-based agencies are controlled research studies. These studies are helpful in identifying factors critical to successful implementation but still lack the practical applications to a complex dynamic system. This article presents a description and preliminary findings of a real-world application of implementation science in community-based agencies, with direct relevance to those targeted by the MHCC strategy. Using a developmental evaluation approach with newly designed implementation supports and tailored strategies to address the unique contexts of implementation projects within the participating agencies has yielded interesting implications for both research and practice.

The PACE program in general provides evidence for the utility of the NIRN frameworks as a useful approach for community-based agencies that are looking to implement the best available evidence on psychotherapy or counselling into their programs. As noted by Barwick, Kimber, and Fearing (2011), the NIRN frameworks lack specificity, so our program supports complemented these frameworks with facilitated sessions, tools, and checklists to enhance understanding and uptake (e.g., Barwick et al., 2010; Danseco, 2013; OCE CYMH, 2013).

The PACE program integrated organizational change management and project management principles, particularly in the planning phase to support implementation efforts. Several implementation frameworks include the measurement of organizational factors such as the implementation climate, learning culture, or leadership yet offer little practical guidance for these types of interventions (e.g., Aarons \& Sommerfeld, 2012; Austin \& Claassen, 2008; Beidas et al., 2013). The PACE program contributes to the few efforts among community-based mental health agencies that integrate organizational level interventions with the implementation process (e.g., Barwick et al., 2011; Williams, Glisson, Hemmelgarn, \& Green, 2016).

The themes that emerged from the analysis of our data demonstrate support for the CFIR constructs and its utility, not just for identifying key factors for implementation research, but also for real-world application of implementation processes. While the CFIR team found no significant alterations to their framework were required (Kirk, Kelley, Yankey, Birken, Abadie, \& Damschroder, 2016), we identified a number of important areas for enhancing the CFIR constructs, particularly for community-based agencies interested in a planned and sustainable approach to implementing the best available evidence. These areas include attention to a broad range of stakeholders, including youth and families who are directly impacted by the EIP, several distinct areas that need to be monitored and evaluated (e.g., fidelity to the practice, implementation capacity, process of implementing, client outcomes), and the processes relating to sustaining and spreading the EIP. These findings are congruent with Ilott and colleagues (2013) who also suggested the addition of more constructs, such as the sustaining and spreading process.

Participating agencies noted the importance of financial resources and supports from the PACE program, as well as their ongoing resource constraints. An important consideration for the implementation of the MHCC strategy on providing appropriate, accessible, coordinated and effective community mental health services will be the extent of comprehensive policy and funding to support these changes. As Raghavan, Bright, and Shadoin (2008) have noted, bridging the research-practice gap in public mental health settings involves 
policy changes with an ecological approach, i.e., efforts at multiple levels by multiple actors. Government policy makers, professional associations, cross-sectoral collaborations, client and family engagement, are some of the other critical elements.

Through the PACE program, we have contributed to the literature by highlighting the organizational and contextual factors beyond clinical training that are part of an ecological approach. Community-based agencies wishing to implement the best available evidence will need to use an implementation science approach that integrates implementation drivers, organizational factors, change management, and project management approaches. This article describes processes that community-based agencies can use to guide their own implementation efforts. Our sector now has sufficient knowledge and research guidance on tools and frameworks for successful large-scale efforts to ensure that mental health outcomes among those we serve continue to improve. Together with the implementation supports of intermediary organizations which can address resource constraints of community-based agencies, implementation science frameworks can bridge the gap between research and sustainable practice change.

\section{REFERENCES}

Aarons, G. A., Hurlburt, M., \& Horwitz, S. M. (2011). Advancing a conceptual model of evidence-based practice implementation in public service sectors. Administration and Policy in Mental Health and Mental Health Services Research, 38(1), 4-23.

Aarons, G. A., \& Sommerfeld, D. H. (2012). Leadership, innovation climate, and attitudes toward evidence-based practice during a statewide implementation. Journal of the American Academy of Child and Adolescent Psychiatry, $51(4), 423-431$.

Active Implementation Hub. (2013). Module 2: Implementation drivers. Available from: http://implementation.fpg. unc.edu/module-2/implementation-drivers

Anderson, M. (2016). Implementation of solution-focused brief therapy. Final Report submitted to the Ontario Centre of Excellence for Child and Youth Mental Health. Retrieved from: http://www.excellenceforchildandyouth.ca/ sites/default/files/gai_attach/PACE-1635_Final_Outcomes_Report.pdf

Austin, M. J., \& Claassen, J. (2008). Implementing evidence-based practice in human service organizations. Journal of Evidence-Based Social Work, 5(1-2), 271-293.

Barratt, M. \& Hodson, R. (2006). Firm foundations: A practical guide to organizational support for the use of research evidence. Dartington, U.K.: Research in Practice.

Barwick, M., Boydell, K., van Dyke, M., \& Worling, J. (2010). E-learning implementation modules. Ottawa, ON: Ontario Centre of Excellence for Child and Youth Mental Health. Retrieved from: http://www.excellenceforchildandyouth.ca/resource-hub/learning-modules

Barwick, M. (2011). Checklist to assess readiness for implementation (CARI). Toronto, ON: Author.

Barwick, M., Kimber, M., \& Fearing, G. (2011). Shifting sands: A case study of process change in scaling up for evidence-based practice. The International Journal of Knowledge, Culture and Change Management, 10(8), 97-114.

Beidas, R. S., Aarons, G., Barg, F., Evans, A., Hadley, T., Hoagwood, K., Marcus, S., Schoewald, S., Walsh, L., \& Mandell, D. S. (2013). Policy to implementation: Evidence-based practice in community mental health study protocol. Implementation Science, 8(1), 1-9.

Botcheva, L., White, C. R., \& Huffman, L. C. (2002). Learning culture and outcomes measurement practices in community agencies. The American Journal of Evaluation, 23(4), 421-434.

Bornbaum, C. C., Kornas, K., Peirson, L., \& Rosella, L. C. (2015). Exploring the function and effectiveness of knowledge brokers as facilitators of knowledge translation in health-related settings: A systematic review and thematic analysis. Implementation Science, 10(1), 1-12.

Brown, J., Rounthwaite, J., \& Barwick, M. (2010). Implementing evidence-based practices: A transformational organizational change process. The International Journal of Knowledge, Culture and Change Management, 10(7), 33-54. 
Charmaz, K. (2006). Constructing grounded theory: A practical guide through qualitative analysis. Thousand Oaks, CA: Sage Publications.

CFIR wiki. (n.d.). Consolidated framework for implementation research technical assistance. Retrieved from: http:// cfirguide.org/index.html

Damschroder, L. J., Aron, D. C., Keith, R. E., Kirsh, S. R., Alexander, J. A., \& Lowery, J. C. (2009). Fostering implementation of health services research findings into practice: A consolidated framework for advancing implementation science. Implementation Science, 4(50). doi:10.1186/1748-5908-4-50

Danseco, E. (2013). Implementation capacity checklist. Ottawa, ON: Ontario Centre of Excellence for Child and Youth Mental Health, Available from E. Danseco, edanseco@cheo.on.ca

Dermenjian, M., Benevides, T., Brear, D., Brouillette, S., Hall, H., Racine, O., Simpson, S., \& Tutt, D. (2016). Leveraging sustainable change with evidence informed practices: Collaborative problem solving. Final report submitted to Ontario Centre of Excellence for Child and Youth Mental Health. Retrieved from: http://www.excellenceforchildandyouth.ca/sites/default/files/gai_attach/PACE-1637_Final_Outcomes_Report.pdf

Dobbins, M., Robeson, P., Ciliska, D., Hanna, S., Cameron, R., O’Mara, L., DeCorby, K., \& Mercer, S. (2009). A description of a knowledge broker role implemented as part of a randomized controlled trial evaluating three knowledge translation strategies. Implementation Science, 4(1), 1-9.

Edmondson, A. C. (2004). Learning from failure in health care: Frequent opportunities, pervasive barriers. Quality and Safety in Health Care, 13(suppl 2), ii3-ii9.

Fixsen, D. L., Naoom, S. F., Blasé, K. A., Friedman, R. M., \& Wallace, F. (2005). Implementation research. A synthesis of the literature. Tampa, FL: University of South Florida, Louis de la Parte Florida Mental Health Institute, The National Implementation Research Network (FMHL Publication \#231).

Glaser, B. G., \& Strauss, A. (1967). The discovery of grounded theory: Strategies for qualitative research. Chicago, IL: Aldine Publishing Co.

Grant, J., Sears, R., Tsagarakis, C., \& Rolfe, R. (2015). Integrating evidence informed approach to service and improving mental health outcomes in school based services using PATTS, and evidence-based program. Final Report submitted to the Ontario Centre of Excellence for Child and Youth Mental Health. Retrieved from: http://www. excellenceforchildandyouth.ca/sites/default/files/gai_attach/PACE-1487_Final_Outcomes_Report.pdf

Hodson, R., \& Cooke, E. (2007). Leading evidence-informed practice: A handbook. Dartington, UK: Research in practice.

Ilott, I., Gerrish, K., Booth, A., \& Field, B. (2013). Testing the consolidated framework for implementation research on health care innovations from South Yorkshire. Journal of Evaluation in Clinical Practice, 19(5), 915-924.

Julian, D. A., Ross, M., \& Partridge, C. (2008). Challenges in supporting community implementation of science-based programs: A critical review of local partnerships for success plans. American Journal of Community Psychology, 41(3), 351-360.

Kerns, S., Rivers, A., \& Enns, G. (2009). Partnerships for success in Washington state: Supporting evidence-based programming for children's mental health. Report on Emotional \& Behavioral Disorders in Youth, 9(3), 53-76.

Kilbourne, A. M., Neumann, M. S., Pincus, H. A., Bauer, M. S., \& Stall, R. (2007). Implementing evidence-based interventions in health care: Application of the replicating effective programs framework. Implementation Science, 2: 42.

Kimber, M., Barwick, M., \& Fearing, G. (2012). Becoming an evidence-based service provider: Staff perceptions and experiences of organizational change. The Journal of Behavioral Health Services \& Research, 39(3), 314-332.

Kirk, M. A., Kelley, C., Yankey, N., Birken, S. A., Abadie, B., \& Damschroder, L. (2016). A systematic review of the use of the consolidated framework for implementation research. Implementation Science, 11(1), 1-13.

Luke D. A., Calhoun A., Robichaux, C. B., Elliott, M. B., \& Moreland-Russell, S. (2014). The program sustainability assessment tool: A new instrument for public health programs. Preventing Chronic Disease 2014;11:130184. doi:http://dx.doi.org/10.5888/pcd11.130184

Lomas, J., Culyer, T., McCutcheon, C., McAuley, L., \& Law, S. (2005). Conceptualizing and combining evidence for health system guidance. Ottawa, ON: Canadian Health Services Research Foundation. Retrieved from: http:// www.cfhi-fcass.ca/migrated/pdf/insightAction/evidence_e.pdf

Mental Health Commission of Canada. (2012). Changing directions, changing lives: The mental health strategy for Canada. Calgary, AB: Author. 
Ontario Centre of Excellence for Child and Youth Mental Health. (2013). Implementing evidence-informed practice: A practical toolkit. Ottawa, ON: Author. Retrieved from: http://www.excellenceforchildandyouth.ca/sites/default/ files/docs/implementation-toolkit.pdf

Ontario Centre of Excellence for Child and Youth Mental Health. (2016). Project showcase: People advancing change through evidence (PACE). Ottawa, ON: Author. Retrieved from: http://www.excellenceforchildandyouth.ca/ resource-hub/project-list-by-type?id=pace

Patton, M. Q. (2010). Developmental evaluation. Applying complexity concepts to enhance innovation and use. New York: Guilford Press.

Phipps, D., \& Morton, S. (2013). Qualities of knowledge brokers: Reflections from practice. Evidence \& Policy: A Journal of Research, Debate and Practice, 9(2), 255-265.

QSR International Pty Ltd. (2010). NVivo qualitative data analysis software, Version 9.

Raghavan, R., Bright, C., \& Shadoin, A. (2008). Toward a policy ecology of implementation of evidence-based practices in public mental health settings. Implementation Science, 3(1), 26.

Schmidt, F. (2015). Community wide implementation and integration of dialectical behavior therapy (DBT) into adult and youth treatment services in Thunder Bay. Final report submitted to the Ontario Centre of Excellence for Child and Youth Mental Health. Retrieved from: http://www.excellenceforchildandyouth.ca/sites/default/files/ gai_attach/PACE-1502_Final_Outcomes_Report.pdf

Schlonsky, A., \& Mildon, R. (2014). Methodological pluralism in the age of evidence-informed practice and policy. Scandinavian Journal of Public Health, 42(13), 18-27.

Starin, A. C., Atkins, M. S., Wehrmann, K. C., Mehta, T., Hesson-McInnis, M. S., Marinez-Lora, A., \& Mehlinger, R. (2014). Moving science into state child and adolescent mental health systems: Illinois' evidence-informed practice initiative. Journal of Clinical Child \& Adolescent Psychology, 43(2), 169-174.

Wandersman, A. (2009). Four keys to success (theory, implementation, evaluation, and resource/system support): High hopes and challenges in participation. American Journal of Community Psychology, 43, 3-21.

Ward, V., House, A., \& Hamer, S. (2009). Knowledge brokering: The missing link in the evidence to action chain? Evidence \& Policy: A Journal of Research, Debate and Practice, 5(3), 267-279.

Williams, N. J., Glisson, C., Hemmelgarn, A., \& Green, P. (2016). Mechanisms of change in the ARC organizational strategy: Increasing mental health clinicians' EBP adoption through improved organizational culture and capacity. Administration and Policy in Mental Health and Mental Health Services Research, 1-15. 